\title{
Degenerate Solutions of General Relativity from Topological Field Theory
}

\author{
John C. Baez \\ Department of Mathematics, University of California \\ Riverside, California 92521 \\ USA \\ email: baez@math.ucr.edu
}

February 24, 1997

\begin{abstract}
Working in the Palatini formalism, we describe a procedure for constructing degenerate solutions of general relativity on 4-manifold $M$ from certain solutions of 2-dimensional $B F$ theory on any framed surface $\Sigma$ embedded in $M$. In these solutions the cotetrad field $e$ (and thus the metric) vanishes outside a neighborhood of $\Sigma$, while inside this neighborhood the connection $A$ and the field $E=e \wedge e$ satisfy the equations of 4-dimensional $B F$ theory. Moreover, there is a correspondence between these solutions and certain solutions of 2-dimensional $B F$ theory on $\Sigma$. Our construction works in any signature and with any value of the cosmological constant. If $M=\mathbb{R} \times S$ for some 3-manifold $S$, at fixed time our solutions typically describe 'flux tubes of area': the 3-metric vanishes outside a collection of thickened links embedded in $S$, while inside these thickened links it is nondegenerate only in the two transverse directions. We comment on the quantization of the theory of solutions of this form and its relation to the loop representation of quantum gravity.
\end{abstract}

\section{Introduction}

Since the introduction by Rovelli and Smolin of spin networks into what used to be called the 'loop representation' of quantum gravity, progress on understanding the kinematical aspects of the theory has been swift [1], 7, 15]. There is a basis of kinematical states given by spin networks, i.e., graphs embedded in space with edges labelled by irreducible representations of SU(2), or spins, and with vertices labelled by intertwining operators. Geometrical observables have been quantized and their matrix elements computed in the spin network basis, giving a concrete - though of course still tentative - picture of 'quantum 3-geometries'. In this picture, the edges of spin networks play the role of quantized flux tubes of area: surfaces acquire area through their intersection with these edges, each edge labelled with spin $j$ contributing an area equal to $\sqrt{j(j+1)}$ times the Planck length squared for each transverse intersection point [2, 16]. 
Unsurprisingly, the dynamical aspects remain more obscure. Thiemann has proposed an explicit formula for the Hamiltonian constraint [17], but this remains controversial, in part because of the difficulty in extracting physical predictions that would test this proposal, or any competing proposals. A crucial problem is the lack of a clear picture of the 4-dimensional, or spacetime, aspects of the theory. For example, diffeomorphism equivalence classes of states annihilated by the Hamiltonian constraint should presumably represent physical states of quantum gravity. Many explicit solutions are known, perhaps the simplest being the 'loop states', corresponding to spin networks with no vertices, or in other words, links with components labelled by spins. However, the interpretation of these states as 'quantum 4-geomeunclear.

In search of some insight into the 4-dimensional interpretation of these loop states, we turn here to classical general relativity. We construct degenerate solutions of the equations of general relativity which at a typical fixed time describe 'flux tubes of area' reminiscent of the loop states of quantum gravity. More precisely, the 3-metric vanishes outside a collection of embedded solid tori, while inside any of these solid tori the metric is degenerate in the longitudinal direction, but nondegenerate in the two transverse directions.

The 4-dimensional picture is as follows: given any surface $\Sigma$ embedded in spacetime, we obtain solutions for which the metric vanishes outside a tubular neighborhood of $\Sigma$. Inside this neighborhood, which we may think of as $\Sigma \times D^{2}$, the 4-metric is degenerate in the two directions tangent to $\Sigma$ but nondegenerate in the two transverse directions. In the 4-geometry defined by one of these solutions, the area of a typical surface $\Sigma^{\prime}$ intersecting $\Sigma$ transversally in isolated points is determined by a sum of contributions from the point in the intersection $\Sigma \cap \Sigma^{\prime}$.

The solutions we consider are inspired by the work of Reisenberger [13], who studied a solution for which the metric vanishes outside a neighborhood of a 2-sphere. Since various formulations of Einstein's equation become inequivalent when extended to the case of degenerate metrics, it is important to say which formulation is being used in any work of this kind. Reisenberger worked in the Plebanski (or self-dual) formalism, and concentrated on the initial-value problem for general relativity extended to possibly degenerate complex metrics, comparing Ashtekar's version of the constraints and a modified version which differs only when the densitized triad field has rank less than 2. Only the latter version admits his '2-sphere solution'. We work 4-dimensionally for the most part, using the classical equations of motion coming from the Palatini formalism, in which the basic fields are a cotetrad field $e$ and a metricpreserving connection $A$ on the 'internal space' bundle. Our work treats arbitrary signatures and arbitrary values of the cosmological constant.

An interesting aspect of our solutions is that they arise from solutions of 2dimensional $B F$ theory, a topological field theory, on the surface $\Sigma$. This takes advantage of the relation between general relativity and $B F$ theory in 4 dimensions, together with the fact that $B F$ theory behaves in a simple manner under dimensional reduction. 
It is also interesting that our construction requires us to choose a thickening of $\Sigma$, that is, to extend the embedding of $\Sigma$ in spacetime to an embedding of $\Sigma \times D^{2}$. The topological data needed to specify a thickening of $\Sigma$ up to diffeomorphism is known as a 'framing' of $\Sigma$. This is precisely the 4-dimensional analog of a framing of a knot or link in a 3-manifold. The possible need for framings in the loop representation of canonical quantum gravity has been widely discussed [5, 9, 12], but here we see it arising quite naturally in the classical context, where the spacetime aspects of framing-dependence are easier to understand.

In the Conclusions we speculate on some of the possible implications of our ideas for the quantum theory. We discuss a 'minisuperspace' model in which one quantizes only the degenerate solutions of general relativity associated to a fixed surface $\Sigma$ in spacetime. We treat this model in detail in another paper [8]; here we only sketch the main ideas. For simplicity we restrict our attention to the Lorentzian signature and certain nonzero values of the cosmological constant $\Lambda$. Our main result is that this minisuperspace model is equivalent to a $G / G$ gauged Wess-Zumino-Witten model on the surface $\Sigma$, with gauge group $\mathrm{SO}(3)$. It follows that if $\Sigma$ intersects space at a given time in some link $L$, states at this time correspond to labellings of the components of $L$ with irreducible representations of quantum $\mathrm{SO}(3)$, or in other words, integral spins

$$
j=0,1,2, \ldots, k / 2
$$

where the 'level' $k$ is an even integer depending on $\Lambda$. In other words, our states are described by a special class of the ' $q$-deformed spin networks' intensively studied by knot theorists [11]. Major and Smolin [12 have suggested using $q$-deformed spin networks to describe states of quantum gravity with nonzero cosmological constant; here we see quite concretely how $q$-deformed spin networks arise in a toy model.

\section{General relativity and $B F$ theory}

Understanding the degenerate solutions of general relativity obtained from topological field theory is perhaps simplest in the Palatini formalism. We begin by recalling some notation introduced in our previous papers [4, 7].

In the Palatini formalism our spacetime $M$ can be any oriented smooth 4-manifold equipped with an oriented vector bundle $\mathcal{T}$ that is isomorphic to $T M$ and equipped with a (nondegenerate) metric $\eta$. In the rest of this section $\eta$ can have any fixed signature. The fiber of $\mathcal{T}$ is called the 'internal space', and $\eta$ is the 'internal metric'. The basic fields are a $\mathcal{T}$-valued 1 -form $e$ on $M$, often called the 'soldering form' or 'cotetrad field', and a metric-preserving connection $A$ on $\mathcal{T}$. Pulling back the internal metric along $e: \mathcal{T} \rightarrow T M$ we obtain a — possibly degenerate - metric $g$ on $M$, given explicitly by

$$
g(v, w)=\eta(e(v), e(w))
$$

The metric $g$ is nondegenerate precisely when $e$ is an isomorphism. 
To write the Palatini action in index-free notation, it is handy to work with differential forms on $M$ taking values in the exterior algebra bundle $\Lambda \mathcal{T}$. In these terms, the Palatini action with cosmological constant $\Lambda$ is given by

$$
S_{\text {Pal }}(A, e)=\int_{M} \operatorname{tr}\left(e \wedge e \wedge F+\frac{\Lambda}{12} e \wedge e \wedge e \wedge e\right)
$$

Here we use the internal metric to regard the curvature $F$ of $A$ as a $\Lambda^{2} \mathcal{T}$-valued 2form on $M$, and use the symbol ' $\wedge$ ' to denote the wedge product of differential forms tensored with the wedge product in $\Lambda \mathcal{T}$. Also, the orientation and internal metric on $\mathcal{T}$ give an 'internal volume form', i.e., a section of $\Lambda^{4} \mathcal{T}$, and thus a map from $\Lambda^{4} \mathcal{T}$-valued forms to ordinary differential forms, which we denote above by ' $\operatorname{tr}$ '.

If we set the variation of this action equal to zero, ignoring boundary terms, we obtain the field equations

$$
d_{A}(e \wedge e)=0, \quad e \wedge\left(F+\frac{\Lambda}{6} e \wedge e\right)=0
$$

where $d_{A}$ denotes the covariant exterior derivative. If $e$ is an isomorphism, the first equation implies that $d_{A} e=0$, which means that the connection on $T M$ corresponding to $A$ via $e$ is torsion-free, and thus equal to the Levi-Civita connection of $g$. The latter equation is then equivalent to Einstein's equation for $g$. Therefore these equations describe an extension of general relativity to the case of degenerate metrics.

Now compare $B F$ theory. This makes sense in any dimension, but has special features in dimension 4 . First let $M$ be any oriented smooth $n$-manifold and let $P$ be a $G$-bundle over $M$, where $G$ is a connected semisimple Lie group. Then the basic fields of the theory are a connection $A$ on $P$ and an $\operatorname{Ad} P$-valued $(n-2)$-form, often called $B$, which we shall call $E$ because of its relation to the gravitational electric field. The action of the theory is

$$
\int_{M} \operatorname{tr}(E \wedge F)
$$

where $F$ is the curvature of $A$ and 'tr' is defined using the Killing form on $\mathfrak{g}$. The corresponding field equations are

$$
d_{A} E=0, \quad F=0 .
$$

In dimension 4 we can also add a cosmological constant term to the action, obtaining

$$
S_{B F}(A, E)=\int_{M} \operatorname{tr}\left(E \wedge F+\frac{\Lambda}{12} E \wedge E\right) .
$$

which gives the field equations

$$
d_{A} E=0, \quad F+\frac{\Lambda}{6} E=0 .
$$


The case $\Lambda \neq 0$ is very different from the case $\Lambda=0$. When $\Lambda \neq 0$, the second equation follows from the first one and the Bianchi identity, so $A$ is arbitrary and it determines $E$. When $\Lambda=0, A$ is flat and $E$ is any solution of $d_{A} E=0$.

We can relate $B F$ theory in 4 dimensions to general relativity in the Palatini formalism if we take $G=\mathrm{SO}(4), \mathrm{SO}(3,1)$, or $\mathrm{SO}(2,2)$, depending on the signature of the internal metric, and we let $P$ be the special orthogonal frame bundle of $\mathcal{T}$. This lets us think of $E$ as a $\Lambda^{2} \mathcal{T}$-valued 2-form, just as $e \wedge e$ is in the Palatini formalism. Comparing the $B F$ theory equations with those of general relativity, it is clear that $e \wedge e$ and $E$ play analogous roles. The easiest way to exploit this is to note that if

$$
E=e \wedge e,
$$

then the equations of $B F$ theory, (四), imply those of general relativity, (2). An analogous observation has been widely remarked on in the context of the Ashtekar formalism (see [i] and the many references therein). Our new observation here is that for (2) to hold, it suffices for (四) to hold where $e$ is nonzero. In what follows we will be interested in degenerate solutions of general relativity where $e$ vanishes everywhere except in a neighborhood of a 2-dimensional surface $\Sigma$, and the equations of 4-dimensional $B F$ theory hold in this neighborhood. We construct these solutions from certain solutions of 2-dimensional $B F$ theory living on $\Sigma$.

\section{Local analysis}

We begin by working locally in coordinates $(t, x, y, z)$ and fixing a local trivialization of $\mathcal{T}$. We consider the surface

$$
\Sigma=\{(t, x, y, z): y=z=0\}
$$

in spacetime, and let

$$
D^{2}=\left\{(y, z): y^{2}+z^{2} \leq r^{2}\right\}
$$

be the disc of radius $r$ in the $y z$ plane. We describe some degenerate solutions of general relativity for which $e$ vanishes outside the neighborhood $\Sigma \times D^{2}$ of the surface $\Sigma$, and the $B F$ theory equations hold inside this neighborhood.

For simplicity, we first consider the case of vanishing cosmological constant. Assume that $A$ is of the form

$$
A=A_{t} d t+A_{x} d x
$$

inside $\Sigma \times D^{2}$, with $A_{t}, A_{x}$ depending only on $t$ and $x$. Let $A$ be arbitrary outside $\Sigma \times D^{2}$. Assume also that $e$ is of form

$$
e=f(y, z)\left[e_{y} d y+e_{z} d z\right]
$$

where $f$ is a smooth function vanishing outside $D^{2}$ and $e_{y}, e_{z}$ depend only on $t$ and $x$. 


\section{Setting}

$$
E=e \wedge e
$$

note that

$$
E=\epsilon f^{2} d y \wedge d z
$$

where

$$
\epsilon=e_{y} \wedge e_{z}
$$

is a $\mathfrak{g}$-valued function on the surface $\Sigma$. (Recall that $\mathfrak{g}$ is the Lie algebra of $G=$ $\mathrm{SO}(4), \mathrm{SO}(3,1)$, or $\mathrm{SO}(2,2)$, depending on the signature we are considering.) Let $\alpha$ denote the restriction of the connection $A$ to $\Sigma$. A calculation then shows that $e$ and $A$ satisfy the equations of general relativity with vanishing cosmological constant:

$$
d_{A}(e \wedge e)=0, \quad e \wedge F=0
$$

if the following equations hold:

$$
d_{\alpha} \epsilon=0, \quad \phi=0,
$$

where $d_{\alpha} \epsilon$ is the exterior covariant derivative of $\epsilon$ with respect to the connection $\alpha$, and $\phi$ is curvature of $\alpha$. These are precisely the equations for 2-dimensional $B F$ theory on the surface $\Sigma$.

However, not every solution of 2-dimensional $B F$ theory on $\Sigma$ gives rise to a degenerate solution of general relativity this way. Starting from any $\alpha, \epsilon$ satisfying (画), we may define the fields $A$ and $E$ as above. These fields satisfy

$$
d_{A} E=0, \quad F=0
$$

inside $\Sigma \times D^{2}$, and $E$ vanishes outside $\Sigma \times D^{2}$. To obtain a solution of general relativity, however, we need a way to write $E$ as $e \wedge e$. There may be no way to do this, or many ways, but we can certainly do it if $\epsilon$ is 'decomposable', that is, if $\epsilon=e_{y} \wedge e_{z}$ for some sections $e_{y}, e_{z}$ of $\mathcal{T}$. Note that since $\epsilon$ is covariantly constant, it will be decomposable if it is of the form $e_{y} \wedge e_{z}$ at any single point of $\Sigma$. We discuss the issue of decomposability further in Section 5 .

If the cosmological constant is nonzero things are a bit more complicated. Suppose again that $\alpha, \epsilon$ satisfy the 2-dimensional $B F$ theory equations (5). We set $E=$ $\epsilon f^{2} d y \wedge d z$ as before, but assume

$$
A=\alpha_{t} d t+\alpha_{x} d x+A_{\perp}
$$

inside $\Sigma \times D^{2}$, with

$$
A_{\perp}=A_{y} d y+A_{z} d z .
$$

Let us show that with an appropriate choice of $A_{\perp}$, the fields $A$ and $E$ satisfy the equations of 4-dimensional $B F$ theory, (4), inside $\Sigma \times D^{2}$. It follows that if $\epsilon$ is 
decomposable, we can write $E=e \wedge e$ and obtain a degenerate solution of the equations of general relativity with cosmological constant term, (2).

To solve equations (4), it suffices to choose $A_{\perp}$ such that

$$
F+\frac{\Lambda}{6} e \wedge e=0
$$

in $\Sigma \times D^{2}$, or in other words,

$$
\begin{aligned}
F_{t x} & =0 \\
F_{t y}, F_{x y}, F_{t z}, F_{x z} & =0 \\
F_{y z} & =-\frac{\Lambda}{6} \epsilon .
\end{aligned}
$$

Equation (6) holds automatically because $\alpha$ is flat. Equations (7) say precisely that the $\mathfrak{g}$-valued functions $A_{y}$ and $A_{z}$ are covariantly constant in the $t$ and $x$ directions. Since $\alpha$ is flat, these equations have a unique solution given any choice of $A_{y}$ and $A_{z}$ on the disc $\{t=x=0\} \times D^{2}$. We can find $A_{y}, A_{z}$ solving (8) on this disc; the solution is not uniquely, but it is unique up to gauge transformations. Choosing any solution and solving equations (7) for $A_{y}$ and $A_{z}$ on the rest of $\Sigma \times D^{2}$, equation (8) then holds throughout $\Sigma \times D^{2}$, because $F_{y z}$ and $\epsilon$ are both covariantly constant in the $t$ and $x$ directions.

In the next section we discuss the global aspects of these solutions of general relativity coming from 2-dimensional $B F$ theory, but first let us examine their physical significance. If we restrict one of our solutions to the slice $t=0$ we obtain the picture shown in Figure 1. Here we have drawn the 'gravitational electric and magnetic fields' $E$ and $B$, by which we mean the restriction of the $E$ and $F$ fields, respectively, to the slice $t=0$. The field $E$ vanishes except in a tube of radius $r$ running along the $x$ axis, so we may think of our solution as describing a 'flux tube' of the gravitational electric field. Since $E$ is a $\mathfrak{g}$-valued 2-form on space, in Figure 1 we have drawn it as small 'surface element' transverse to the $x$ axis. Outside the flux tube the gravitational magnetic field is arbitrary, but inside it we have

$$
B=-\frac{\Lambda}{6} E
$$

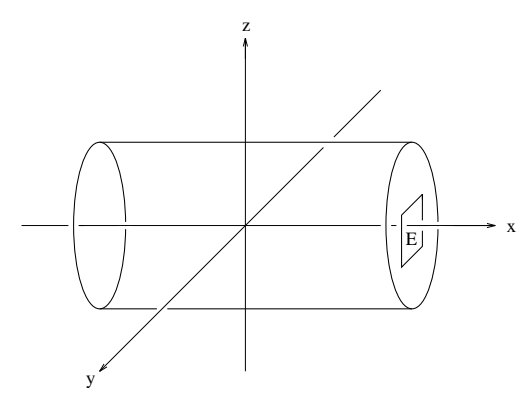

1. Flux tube of gravitational electric field 
The 3-metric is zero outside this flux tube, while inside, the 3-metric is degenerate in the $x$ direction but nondegenerate in the two transverse directions. In the 3geometry defined by this solution, the area of any surface is zero unless it intersects the flux tube. We see therefore that this solution is a kind of classical analog of the 'loop states' of quantum gravity, which have been shown to represent quantized flux tubes of area [2, 16]. We make this analogy more precise in the next section, where we consider flux tube solutions associated to arbitrary thickened links in space.

The case of nonzero cosmological constant is particularly interesting. Then $B_{y z}$ is typically nonzero in the flux tube, so the connection $A$ will typically have nontrivial holonomy around a small loop wrapping around the tube. Figure 2 shows a circular loop $\gamma_{x}$ of radius $r$ centered at the point $x$ on the $x$ axis. To define the holonomy around such a loop as an element of the gauge group $G$, rather than a mere conjugacy class, we need to choose a basepoint for the loop. We can do this using a 'framing' of the flux tube, or more precisely, a curve running along the surface of the tube that intersects each loop $\gamma_{x}$ at one point, which we take as the basepoint. In Figure 2 we have chosen as our framing the line $z=0, y=-r$. Using this framing we may define the holonomy

$$
g(x)=T \exp \oint_{\gamma_{x}} A .
$$

Using the definition of parallel transport we easily see that $g(x)$ is covariantly constant along the flux tube:

$$
\partial_{x} g+\left[A_{x}, g\right]=0 .
$$

In the spacetime picture, $g$ becomes a function of $t$ and $x$, and one can also check that

$$
\partial_{t} g+\left[A_{t}, g\right]=0
$$

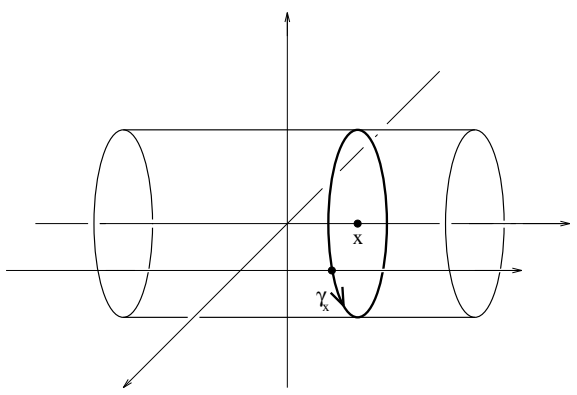

2. Holonomy of connection around flux tube

In the next section we show that in general, we need to equip a surface $\Sigma$ with the 4-dimensional analogue of a framing in order to obtain solutions of general relativity from solutions of 2-dimensional $B F$ theory on $\Sigma$. This extra structure also lets us define a field $g$ on $\Sigma$ that measures the holonomy of $A$ around small loops around $\Sigma$. As above, this field $g$ will be covariantly constant on $\Sigma$. In fact, this field is 
closely related to the group-valued field in the $G / G$ gauged WZW model on $\Sigma$ that we describe in the Conclusions.

\section{Global analysis}

In this section we extend the local analysis of the previous section to show that for any framed surface embedded in spacetime, we can obtain degenerate solutions of general relativity from certain solutions of 2-dimensional $B F$ theory on this surface. Let $M$ be an oriented 4-manifold, and let $\Sigma$ be a 2-manifold embedded in $M$. As before let $\mathcal{T}$ be a vector bundle isomorphic to $T M$ equipped with a nondegenerate metric of some fixed signature, and let $P \rightarrow M$ be the special orthogonal frame bundle of $\mathcal{T}$.

By a 'framing' of $\Sigma$ we mean a homotopy class of trivializations of its normal bundle. We call an extension of the embedding

$$
i: \Sigma \hookrightarrow M
$$

to an embedding

$$
j: \Sigma \times D^{2} \hookrightarrow M
$$

a 'thickening' of $\Sigma$. Any thickening determines a framing in a natural way. In fact, any framing comes from some thickening in this way. Moreover, two thickenings $j, j^{\prime}$ determine the same framing if and only if they are ambient isotopic, that is, if $j^{\prime}=f j$ for some diffeomorphism of $M$ connected to the identity. These results can be proved just as the analogous results were proved for thickenings of framed links [5].

In what follows we assume $\Sigma$ is equipped with a framing, and we pick an arbitrary thickening compatible with this framing. Using this thickening we think of $\Sigma \times D^{2}$ as a submanifold of $M$. We assume $\alpha$ is a connection on $\left.P\right|_{\Sigma}$ and $\epsilon$ is a section of $\operatorname{Ad} P$ over $\Sigma$, and we assume that these fields satisfy the equations of 2 -dimensional $B F$ theory,

$$
d_{\alpha} \epsilon=0, \quad \phi=0
$$

where $\phi$ is the curvature of $\alpha$. From this data we shall construct a connection $A$ on $P$ and an $\operatorname{Ad} P$-valued 2-form $E$ on $M$ such that $E$ is zero outside the thickening $\Sigma \times D^{2} \subseteq M$, and the equations of 4-dimensional $B F$ theory, (4), hold inside $\Sigma \times D^{2}$.

First we construct the field $E$ as follows. Using notation similar that of the previous section, let $d y \wedge d z$ denote the standard volume form on $D^{2}$ and let $f$ be a smooth function on $D^{2}$ vanishing near the boundary. Pulling back the 2-form $f^{2} d y \wedge d z$ from $D^{2}$ to $\Sigma \times D^{2}$ along the projection $p_{2}: \Sigma \times D^{2} \rightarrow D^{2}$, we obtain a 2-form on $\Sigma \times D^{2}$ which by abuse of language we again denote by $f^{2} d y \wedge d z$.

We can choose a metric-preserving isomorphism between the bundle $\left.\mathcal{T}\right|_{\Sigma \times D^{2}}$ and the bundle $\left.p_{1}^{*} T\right|_{\Sigma}$, where $p_{1}: \Sigma \times D^{2} \rightarrow \Sigma$ is the projection onto the first factor. Requiring that this isomorphism be the identity for points in $\Sigma$, it is then unique up to gauge transformation. We fix such an isomorphism. Using this, the pullback $p_{1}^{*} \epsilon$ 
can be thought of as a section of $\operatorname{Ad} P$ on $\Sigma \times D^{2}$. We then define the $\operatorname{Ad} P$-valued 2-form $E$ by

$$
E=\epsilon f^{2} d y \wedge d z
$$

on $\Sigma \times D^{2}$, and define $E$ to be zero on the rest of $M$.

Next we construct the connection $A$ on $P$. We pull back $\alpha$ to a connection on $\left.p_{1}^{*} P\right|_{\Sigma}$, and using the isomorphism chosen in the previous paragraph we think of this as a connection on $\left.P\right|_{\Sigma \times D^{2}}$, which we extend arbitrarily to a connection $A_{\|}$on all of $P$. We define $A$ by

$$
A=A_{\|}+A_{\perp}
$$

where $A_{\perp}$ is an $\operatorname{Ad} P$-valued 1-form given as follows. Inside $\Sigma \times D^{2}$ we set

$$
A_{\perp}=A_{y} d y+A_{z} d z
$$

where $A_{z}=0$ and for any point $(p, y, z) \in \Sigma \times D^{2}$,

$$
A_{y}(p, y, z)=\frac{\Lambda}{6} \int_{0}^{z} \epsilon\left(p, y, z^{\prime}\right) f^{2}\left(y, z^{\prime}\right) d z^{\prime}
$$

Outside $\Sigma \times D^{2}$, we let $A_{\perp}$ be arbitrary.

Finally, we check that the equations of 4-dimensional $B F$ theory hold in $\Sigma \times D^{2}$. When $\Lambda \neq 0$ we only need to check that $F=-\frac{\Lambda}{6} E$. We work using local coordinates $t, x$ on $\Sigma$. Our choice of $A_{\perp}$ ensures that

$$
F_{y z}=-\frac{\Lambda}{6} \epsilon f^{2}
$$

so $F_{y z}=-\Lambda E_{y z} / 6$. We have $E_{t x}=0$ by construction and $F_{t x}=0$ since $A_{\|}$is flat. We have $E_{t y}=0$ by construction, and at any point $(p, y, z) \in \Sigma \times D^{2}$ we have

$$
\begin{aligned}
F_{t y} & =\partial_{t} A_{y}+\left[A_{t}, A_{y}\right] \\
& =\frac{\Lambda}{6} \int_{0}^{z}\left(\partial_{t} \epsilon\left(p, y, z^{\prime}\right)+\left[\alpha_{t}(p), \epsilon\left(p, y, z^{\prime}\right)\right]\right) f^{2}\left(y, z^{\prime}\right) d z^{\prime} \\
& =0
\end{aligned}
$$

since $d_{\alpha} \epsilon=0$. Similarly we have $E_{t z}=E_{x y}=E_{x z}=0$ and $F_{t z}=F_{x y}=F_{x z}=0$. When $\Lambda=0$ we also need to check that $d_{A} E=0$ in $\Sigma \times D^{2}$. This follows from $d_{\alpha} \epsilon=0$.

Summarizing, we have:

Theorem 1. Let $\Sigma$ be a framed 2-manifold embedded in a 4-manifold $M$, and let $\mathcal{T}$ be a vector bundle over $M$ isomorphic to the tangent bundle and equipped with a metric $\eta$ of arbitrary fixed signature. Let $P$ be the special orthogonal frame bundle of $\mathcal{T}$. For any value of $\Lambda$, there is a natural map from solutions $(\alpha, \epsilon)$ of 2-dimensional $B F$ theory on $\Sigma$ (where $\alpha$ is a connection on $\left.P\right|_{\Sigma}$ and $\epsilon$ is a section of $\left.\operatorname{Ad} P\right|_{\Sigma}$ ) to 
gauge and diffeomorphism equivalence classes of fields $(A, E)$ (where $A$ is a connection on $P$ and $e$ is a $\operatorname{Ad} P$-valued 2-form on $M$ ) satisfying the equations of 4-dimensional $B F$ theory in a neighborhood of $\Sigma$. Given any of these solutions, we may extend $A$ and $E$ smoothly to all of $M$ with $E$ vanishing outside the given neighborhood of $\Sigma$.

Here the term 'natural' refers to the fact that given a diffeomorphism $F: M \rightarrow M^{\prime}$ mapping the framed surface $\Sigma \subseteq M$ to the framed surface $\Sigma^{\prime} \subseteq M^{\prime}$ and carrying the solution $(\alpha, \epsilon)$ of 2-dimensional $B F$ theory on $\Sigma$ to the solution $\left(\alpha^{\prime}, \epsilon^{\prime}\right)$ on $\Sigma^{\prime}$, the corresponding solution $(A, E)$ of 4-dimensional $B F$ theory on a neighborhood of $\Sigma$ is mapped to the corresponding solution $\left(A^{\prime}, E^{\prime}\right)$ on a neighborhood of $\Sigma^{\prime}$, up to diffeomorphism and gauge transformation (or more precisely, up to a bundle automorphism).

\section{Decomposability}

Given the hypotheses of Theorem 1, if $\epsilon$ is decomposable at one point of each component of the surface $\Sigma$, it is automatically decomposable throughout $\Sigma$. We may thus write $E=e \wedge e$ and obtain a degenerate solution of the equations of general relativity, (目), on $M$.

One way to guarantee decomposability is to require that $(\alpha, \epsilon)$ is actually a solution of $\mathrm{SO}(3) B F$ theory on $\Sigma$. Here we restrict attention to the signatures ++++ and +++- , so that $G$ is either $\mathrm{SO}(4)$ or $\mathrm{SO}(3,1)$, and we use the fact that $\mathrm{SO}(3)$ is a subgroup of these groups. Suppose we split $\left.\mathcal{T}\right|_{\Sigma}$ into a line bundle and a 3dimensional vector bundle on which the internal metric $\eta$ is positive definite. Using a metric-preserving isomorphism between $\left.\mathcal{T}\right|_{\Sigma \times D^{2}}$ and $\left.p_{1}^{*} \mathcal{T}\right|_{\Sigma}$, we obtain a splitting

$$
\mathcal{T}_{\Sigma \times D^{2}}=L \oplus \oplus^{3} \mathcal{T}
$$

where $\eta$ is positive definite on ${ }^{3} \mathcal{T}$. Let ${ }^{3} P$ denote the special orthogonal frame bundle of ${ }^{3} \mathcal{T}$. This is a principal $\mathrm{SO}(3)$-bundle, which we may think of as a sub-bundle of the principal $G$-bundle $\left.P\right|_{\Sigma \times D^{2}}$. Similarly, the vector bundle $\operatorname{Ad}^{3} P$ is a sub-bundle of the vector bundle $\left.\operatorname{Ad} P\right|_{\Sigma \times D^{2}}$. Locally, we may think of sections of the former bundle as $\mathfrak{s o}(3)$-valued functions, and sections of the latter as $\mathfrak{g}$-valued functions, where $\mathfrak{g}$ is either $\mathfrak{s o}(4)$ or $\mathfrak{s o}(3,1)$.

Suppose we have a solution of 2-dimensional $B F$ theory on $\Sigma$ for which the connection $\alpha$ reduces to a connection on ${ }^{3} P$ and the section $\epsilon$ of $\operatorname{Ad} P$ is actually a section of $\operatorname{Ad}^{3} P$. Then $\epsilon$ is automatically decomposable, since every vector $u \in \mathbb{R}^{3}$ is the cross product of two other vectors. Moreover, there is a canonical way to decompose $\epsilon$, since we can always write $u=v \times w$ where $v$ and $w$ have the same length and $u, v, w$ form a right-handed triple. The map $u \mapsto(v, w)$ is not smooth at $u=0$, but if $\epsilon$ is zero at some point of $\Sigma$ it is zero throughout that component of $\Sigma$, so we indeed have a canonical way to write

$$
\epsilon=e_{y} \wedge e_{z}
$$


for smooth sections $e_{y}, e_{z}$ of ${ }^{3} \mathcal{T}$.

We thus obtain the following:

Theorem 2. Given the hypotheses of Theorem 1, assume that the signature of $\eta$ is either ++++ or +++- , and assume $\left.\mathcal{T}\right|_{\Sigma}$ is equipped with a 3-dimensional subbundle on which $\eta$ is positive definite. For any value of $\Lambda$, there is a natural map from solutions $(\alpha, \epsilon)$ of 2-dimensional $\mathrm{SO}(3)$ BF theory on $\Sigma$ (where $\alpha$ is a connection on $\left.{ }^{3} P\right|_{\Sigma}$ and $\epsilon$ is a section of $\left.\left.\mathrm{Ad}^{3} P\right|_{\Sigma}\right)$ to gauge and diffeomorphism equivalence classes of fields $(A, e)$ (where $A$ is a connection on $P$ and $e$ is an $\mathcal{T}$-valued 1-form on $M$ ) satisfying the equations of general relativity on $M$.

One may wonder to what extent these solutions of general relativity depend on our choice of 3-dimensional sub-bundle of $\left.\mathcal{T}\right|_{\Sigma}$, and whether such a choice always exists. At least in the case of signature ++++ , these issues work out nicely. In this case, we can always choose a splitting of $\mathcal{T}_{\Sigma}$ into a line bundle $L$ and a 3-dimensional vector bundle $M$, and this splitting is unique up to a small gauge transformation. It follows that our map from solutions $(\alpha, \epsilon)$ to gauge and diffeomorphism equivalence classes of solutions $(A, e)$ is independent of the choice of 3-dimensional sub-bundle of $\left.\mathcal{T}\right|_{\Sigma}$, as long as we choose it so that its orthogonal complement is trivial.

To see these facts we need a little homotopy theory. Note that a splitting $\mathcal{T}_{\Sigma}=$ $L \oplus M$ is equivalent to a reduction of structure group from $\mathrm{SO}(4)$ to $\mathrm{SO}(3)$. This is also equivalent to a lifting

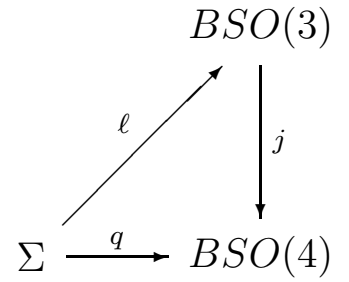

where $q$ is the classifying map of the bundle $\left.P\right|_{\Sigma}$, and $j$ is the map between classifying spaces induced by the inclusion $\mathrm{SO}(3) \hookrightarrow \mathrm{SO}(4)$. Homotopy classes of such liftings correspond to homotopy classes of maps from $\Sigma$ to $S O(4) / S O(3) \cong S^{3}$, which is the homotopy fiber of the map $j$. However, all maps from $\Sigma$ to $S^{3}$ are homotopic, so any two liftings are homotopic. Thus, suppose we are given two splittings, $\mathcal{T}_{\Sigma}=L \oplus M$ and $\mathcal{T}_{\Sigma}=L^{\prime} \oplus M^{\prime}$. These correspond to two liftings $\ell$ and $\ell^{\prime}$, and since there exists a homotopy between $\ell$ and $\ell^{\prime}$, there exists a continuous one-parameter family of splittings interpolating between the two given ones. It follows that there is a small gauge transformation carrying $L$ to $L^{\prime}$ and $M$ to $M^{\prime}$.

\section{Conclusions}

Despite many parallels, the relevance - if any — of our 'flux tube' solutions to the loop representation of quantum gravity is not yet clear. Perhaps, however, we can 
begin to understand this by quantizing the theory of solutions of this sort associated to a fixed surface in spacetime. We can think of this as an unusual sort of 'minisuperspace model'. In such a model all but finitely many degrees of freedom of the classical gravitational field are ignored, and then the rest are quantized. Presumably any such model is only a caricature of quantum gravity. At best we can hope that, like a good caricature, it reveals certain interesting features of the subject.

Here we give a rough outline of how this might proceed, leaving a more careful treatment to another paper [8]. We follow a path-integral approach, considering only the case of nonzero cosmological constant. Let $M$ be an oriented 4-manifold and let $\mathcal{T}$ be a bundle over $M$ isomorphic to the tangent bundle and equipped with a Lorentzian metric $\eta$. Fix a compact framed surface $\Sigma$ in $M$, a thickening of $\Sigma$, and a 3-dimensional sub-bundle ${ }^{3} \mathcal{T}$ of $\left.\mathcal{T}\right|_{\Sigma \times D^{2}}$ as in the previous section. Working in the Palatini formalism, we integrate only over field configurations $(A, e)$ for which:

1. Inside $\Sigma \times D^{2}, A$ reduces to a connection on ${ }^{3} \mathcal{T}$ and $F+\frac{\Lambda}{6} e \wedge e=0$.

2. Outside $\Sigma \times D^{2}, A$ is arbitrary and $e=0$.

For such field configurations the action can be expressed purely in terms of $A$ :

$$
\begin{aligned}
S_{\text {Pal }}(A, e) & =\int_{M} \operatorname{tr}\left(e \wedge e \wedge F+\frac{\Lambda}{12} e \wedge e \wedge e \wedge e\right) \\
& =-\frac{3}{\Lambda} \int_{\Sigma \times D^{2}} \operatorname{tr}(F \wedge F)
\end{aligned}
$$

If we fix a connection $A_{0}$ of the above form that is flat on the boundary $\Sigma \times S^{1}$ of the thickening, and use this to think of $A$ as an $\operatorname{Ad} P$-valued 1-form, we obtain by Stokes' theorem

$$
S_{P a l}(A, e)=-\frac{3}{\Lambda} S_{C S}(A)+c
$$

where $c$ depends only on the fixed connection $A_{0}$ and $S_{C S}(A)$ is the Chern-Simons action

$$
S_{C S}(A)=\int_{\Sigma \times S^{1}} \operatorname{tr}\left(A \wedge d A+\frac{2}{3} A \wedge A \wedge A\right)
$$

Now we are in a position to take full advantage of the following 'ladder of field theories' [4 in dimensions 2, 3, and 4: 


\section{4d: $\quad$ General relativity $\rightarrow B F$ theory $\rightarrow$ Chern theory}

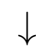

3d:

2d:
Chern-Simons theory

$\downarrow$

As we have seen, for any framed surface $\Sigma$ in spacetime, general relativity has a class of degenerate solutions coming from 4-dimensional $B F$ theory. Our minisuperspace model amounts to 4-dimensional $B F$ theory on the thickened surface $\Sigma \times D^{2}$. Using the equations of motion of $B F$ theory to write the action purely as a function of $F$, it is then proportional to the second Chern number

$$
c_{2}=\frac{1}{8 \pi^{2}} \int_{M} \operatorname{tr}(F \wedge F) \text {. }
$$

This action gives a theory involving only the $A$ field, which we may call 'Chern theory'. The Lagrangian of Chern theory is a closed 4-form, so it has no bulk degrees of freedom, and on $\Sigma \times D^{2}$ it reduces to Chern-Simons theory on $\Sigma \times S^{1}$.

The final step down the dimensional ladder uses the fact that Chern-Simons theory on a 3-manifold of form $\Sigma \times S^{1}$ is equivalent to the $G / G$-gauged Wess-Zumino-Witten model on $\Sigma$. This theory is a 2-dimensional topological field theory having a basis of states corresponding to irreducible representations of the quantum group associated to the gauge group $G$, which in our case is $\mathrm{SO}(3)$. We see therefore that in the canonical picture, when $\Sigma$ intersects space a given time in some link $L$, states of our minisuperspace model are described to labellings of the components of $L$ with irreducible representations of quantum $\mathrm{SO}(3)$, or in other words, integral spins $j=$ $0,1,2, \ldots, k / 2$ where the 'level' $k$ is an even integer depending on $\Lambda$. A link labelled this way is simply a $q$-deformed spin network with no vertices.

While we have included the Wess-Zumino-Witten model in our picture of the ladder of field theories, it has only an indirect relevance to our minisuperspace model. It is a conformal field theory whose conformal blocks form a basis of states of the $G / G$ gauged Wess-Zumino-Witten model. In string theory, the string worldsheet is equipped with a conformal structure, and some of the transverse vibrational modes of the string are described by Wess-Zumino-Witten models living on this surface. In the minisuperspace model sketched above, we see instead the $G / G$ gauged Wess-ZuminoWitten model living on the surface $\Sigma$. There is, of course, no opportunity for us to see a conformal field theory on $\Sigma$, because this surface is not equipped with a metric; even in any particular solution the metric is only nondegenerate in the two directions transverse to $\Sigma$. 
This begins to make more precise our previous speculation that the loop representation of quantum gravity is related to background-free string theory, with the loops arising as $t=0$ slices of string worldsheets [3]. It is intriguing that in a somewhat different framework, Jacobson [10] has constructed degenerate solutions of general relativity in which the metric is nondegenerate in the directions tangent to a surface, and by this means obtains a conformal field theory. Comparing his solutions and ours may shed more light on the relation between the loop representation of quantum gravity and string theory — or at least 2-dimensional field theory.

\section{Acknowledgements}

I would like to thank Ted Jacobson for getting me interested in degenerate solutions of general relativity coming from field theories on surfaces, Mike Reisenberger for pointing out that his '2-sphere solution' of general relativity should be related to $B F$ theory, Jorge Pullin for emphasizing the importance of decomposability of the $E$ field, and W. Dale Hall and Clarence Wilkerson for helping me with the homotopy theory. I would also like to thank the Erwin Schrödinger Institute in Vienna, the Theoretical Physics Group at Imperial College, and the Center for Gravitational Physics and Geometry at Pennsylvania State University for their hospitality while this work was being done.

\section{References}

[1] Abhay Ashtekar, Jerzy Lewandowski, Donald Marolf, Jose Mourao, and Thomas Thiemann, Quantization of diffeomorphism invariant theories of connections with local degrees of freedom, J. Math. Phys. 36 (1995), 6456-6493.

[2] A. Ashtekar and J. Lewandowski, Quantum Theory of Geometry I: Area Operators, Class. Quantum Grav. 14 (1997), A55-A81.

[3] J. Baez, Strings, loops, knots and gauge fields, in Knots and Quantum Gravity, ed. J. Baez, Oxford U. Press, Oxford, 1994.

[4] J. Baez, Knots and quantum gravity: progress and prospects, to appear in the Proceedings of the Seventh Marcel Grossman Meeting on General Relativity, gr-qc/9410018.

[5] J. Baez, Link invariants, holonomy algebras and functional integration, Jour. Funct. Anal. 127 (1995), 108-131.

[6] J. Baez, Four-dimensional BF theory as a topological quantum field theory, Lett. Math. Phys. 38 (1996), 129-143. 
[7] J. Baez, Spin networks in nonperturbative quantum gravity, in The Interface of Knots and Physics, ed. Louis Kauffman, American Mathematical Society, Providence, Rhode Island, 1996.

[8] J. Baez, BF theory and quantum gravity in four dimensions, manuscript in preparation.

[9] C. Di Bartolo, R. Gambini, J. Griego, J. Pullin, The space of states of quantum gravity in terms of loops and extended loops: some remarks, Jour. Math. Phys. 36 (1995), 6511.

[10] T. Jacobson, $1+1$ sector of 3+1 gravity, Class. Quant. Grav. 13 (1996), L1-L6.

[11] L. Kauffman and S. Lins, Temperley-Lieb Recoupling Theory and Invariants of 3-Manifolds, Princeton U. Press, Princeton, New Jersey, 1994.

[12] S. Major, L. Smolin, Quantum deformation of quantum gravity, Nucl. Phys. B473 (1996), 267-290.

[13] M. Reisenberger, New constraints for canonical general relativity, Nucl. Phys. B457 (1995), 643-687.

[14] M. Reisenberger and C. Rovelli, "Sum over surfaces" form of loop quantum gravity, preprint available as gr-qc/9612035.

[15] C. Rovelli and L. Smolin, Spin networks in quantum gravity, Phys. Rev. D52 (1995), 5743-5759.

[16] C. Rovelli and L. Smolin, Discreteness of area and volume in quantum gravity, Nucl. Phys. B442 (1995), 593-622. Erratum, ibid. B456 (1995), 753.

[17] T. Thiemann, Anomaly-free formulation of non-perturbative, four-dimensional Lorentzian quantum gravity, Phys. Lett. B380 (1996), 257-264. 